\section{OP0009 DERIVATION AND VALIDATION OF THE SCLERODERMA LUNG 3-STAGE INDEX (SL3SI), A NEW FUNCTIONAL INDEX FOR INTERSTITIAL LUNG DISEASE WITH PROGNOSTIC IMPLICATIONS}

A. Santaniello ${ }^{1}$, C. Bellocchi ${ }^{1}$, L. Bettolini ${ }^{1}$, M. Cassavia ${ }^{1}$, G. Montanelli ${ }^{1}$, A. Severino ${ }^{1}$, M. Caronni ${ }^{1}$, C. Campochiaro ${ }^{2}$, E. De Lorenzis ${ }^{3,4}$, G. Natalello ${ }^{3,4}$ P. Delvino ${ }^{5}$, C. Tirelli ${ }^{5}$, L. Cavagna ${ }^{5}$, G. De Luca ${ }^{2}$, S. L. Bosello ${ }^{3}$, L. Beretta ${ }^{1}$. ${ }^{1}$ Scleroderma Unit, Referral Center for Systemic Autoimmune Diseases, Fondazione IRCCS Ca' Granda Ospedale Maggiore Policlinico di Milano, Milan, Italy; ${ }^{2}$ Unit of Immunology, Rheumatology, Allergy and Rare Diseases IRCCS San Raffaele Hospital, Università Vita-Salute San Raffaele, Milan, Italy; ${ }^{3}$ Rheumatology Division, Fondazione Policlinico Universitario A. Gemelli IRCCS, Rome, Italy; ${ }^{4}$ Catholic University of the Sacred Heart, Rome, Italy; ${ }^{5}$ Division of Rheumatology, University and IRCCS Policlinico S. Matteo Foundation, Pavia, Italy

Background: The staging of interstitial lung disease (ILD) is important to monitor disease progression and for prognostication. A disease severity scale of Systemic Sclerosis (SSc)-related lung disease has long been proposed (i.e. Medsger's severity scale). This scale was mostly developed by discussion and consensus and stage thresholds were not computed by a data-driven approach. Hidden Markov models (HMM) are methods to estimate population quantities for chronic diseases with a staged interpretation which are diagnosed by markers measured at irregular intervals.

Objectives: To build a SSc-ILD specific disease severity scale with prognostic relevance via HMM modeling.

Methods: A total of $358 \mathrm{SSc}$ patients at risk for or with ILD were enrolled in a discovery (207 cases, Milan1) and in a validation (151 cases, Milan2, Pavia and Rome) cohort. Patients were included if satisfied the following criteria: 1) Diagnosis of SSc according to the EULAR/ACR 2013 criteria, 2) absence of anticentromere antibodies, 3) dcSSc subset or 4) other subsets with either 4a) ILD-related antibodies (Scl70, PmScl, Ku) or 4b) evidence of ILD on HRCT, 5) disease duration $<5$ years at the time of the first pulmonary function test (PFT). Serial PFTs were retrieved and the time up to the last available visit -if the patient alive-, or to death due to pulmonary complications, was recorded. HMM were used to estimate the threshold of a 3-stage model (SL3SI, Scleroderma Lung 3-Stage Index) based on PFT functional values (normal/mild, moderate, severe involvement) in the discovery cohort. Survival estimates of the SL3SI model were compared to Medsger's severity classes estimates and their predictive capability evaluated via the explained residual variation $\left(R^{2}\right)$ of prediction errors (the higher the better). One-hundred random replicates were generated to simulate the prediction effort in patients with different disease duration and lung severity.

Results: Patients characteristics are summarized in the Table. Fifteen-years survival estimates for Mesdger's classes in the discovery set were: normal $=0.88$, mild $=0.86$, moderate $=0.84$ and severe $=0.71$. The SL3SI was defined by the following thresholds: normal/mild, FVC and DLco $>=75 \%$; moderate FVC or DLco 74-55\%; severe, FVC or DLco $<55 \%$. SL3SI 15-yrs survival estimates were: nor$\mathrm{mal} / \mathrm{mild}=0.89$, moderate $=0.82$ and severe $=0.63$. Prediction analysis showed a higher $\mathrm{R}^{2}$ values at $15 \mathrm{yrs}$ for the SL3SI compared to Medsger's classes, providing evidence for a better predictive capability of the former (discovery: 0.31 vs 0.25 ; validation: 0.28 vs 0.19 ).

Conclusion: The SL3SI, a simplified 3-stage functional model of SSc-ILD, yields better survival estimates and long-term prognostic information than Medsger's classes. Its reproducibility and ease of use make it a useful tool for the functional and prognostic evaluation of SSc patients at risk for or with ILD.

Table:

\begin{tabular}{lcc}
\hline Variables & Discovery $(\mathrm{n}=207)$ & Replication $(\mathrm{n}=151)$ \\
\hline DcSSc & $62(30 \%)$ & $98(64 \%)$ \\
Age at first PFR & $48.6 \pm 12$ & $49.1 \pm 14.4$ \\
Disease duration at first PFR & $1.7 \pm 1.6$ & $1.3 \pm 2.4$ \\
FVC & $90.5 \pm 18.1$ & $91.1 \pm 20.2$ \\
DLco & $70.7 \pm 19.8$ & $61.3 \pm 20.1$ \\
ILD on HRCT & $179(86 \%)$ & $125(80 \%)$ \\
Scl70 & $157(76 \%)$ & $153(78 \%)$ \\
SSA & $63(30 \%)$ & $32(21 \%)$ \\
n of visits & 3857 & 1473 \\
Follow-up time, yrs & $11 \pm 5.6$ & $10.6 \pm 5.7$ \\
Deaths & $27(13 \%)$ & $23(15 \%)$ \\
\hline
\end{tabular}

Disclosure of Interests: Alessandro Santaniello: None declared, Chiara Bellocchi: None declared, Luca Bettolini: None declared, Marcello Cassavia: None declared, Gaia Montanelli: None declared, Adriana Severino: None declared, Monica Caronni: None declared, Corrado Campochiaro Speakers bureau: Novartis, Pfizer, Roche, GSK, SOBI, Enrico De Lorenzis: None declared, Gerlando Natalello: None declared, Paolo Delvino: None declared, Claudio Tirelli: None declared, Lorenzo Cavagna: None declared, Giacomo De Luca Speakers bureau: SOBI, Novartis, Celgene, Pfizer, MSD, Silvia Laura Bosello: None declared, Lorenzo Beretta Grant/research support from: Pfizer

DOI: 10.1136/annrheumdis-2020-eular.5788

\section{Opening Plenary abstract session}

\begin{tabular}{|l|l}
\hline OP0010 & EFFECTIVENESS OF THE MAKING IT WORK TM \\
PROGRAM AT IMPROVING PRESENTEEISM \\
AND WORK CESSATION IN WORKERS WITH \\
INFLAMMATORY ARTHRITIS - RESULTS OF A \\
RANDOMIZED CONTROLLED TRIAL
\end{tabular}

A. Luquini ${ }^{1,2}$, Y. Zheng ${ }^{2}$, H. Xie, ${ }^{2,3}$, C. Backman ${ }^{1,2}$, P. Rogers ${ }^{2}$, A. Kwok ${ }^{2}$, A. Knight ${ }^{2}$, M. Gignac ${ }^{4}$, D. Mosher ${ }^{5}$, L. Li ${ }^{1,2}$, J. Esdaile ${ }^{1,2}$, C. Thorne ${ }^{6}$, D. Lacaille ${ }^{1,2} .{ }^{1}$ The University of British Columbia, Vancouver, Canada; ${ }^{2}$ Arthritis Research Canada, Richmond, Canada; ${ }^{3}$ Simon Fraser University, Faculty of Health Sciences, Burnaby, Canada; ${ }^{4}$ Institute for Work and Health - University of Toronto, Dalla Lana School of Public Health, Toronto, Canada; ${ }^{5}$ Cumming School of Medicine, University of Calgary, Department of Medicine, Division of Rheumatology, Calgary, Canada; ${ }^{6}$ University of Toronto, Department of Medicine, Division of Rheumatology, Toronto, Canada

Background: Arthritis often leads to presenteeism (decreased at-work productivity), missed days from work and permanent work disability, leading to reduced quality of life and high costs to individuals and society. Yet, health services addressing the employment needs of people with arthritis are lacking.

Objectives: We evaluated the effectiveness of the Making-it-Work ${ }^{\mathrm{TM}}$ (MiW) program, an online self-management program developed to help people with inflammatory arthritis $(\mathrm{IA})$ deal with employment issues.

Methods: A multi-center RCT evaluated the effectiveness of MiW at improving presenteeism and preventing work cessation (WC) over two years. Participants were recruited from rheumatologist practices, consumer organizations and arthritis programs, in three Canadian provinces. Eligibility criteria: diagnosis of IA, employed, age 18-59, and concerned about ability to work. Participants were randomized 1:1 to MiW or usual care plus printed material on workplace tips. MiW consists of five online self-learning modules and group meetings, and individual vocational counselling and ergonomic consultations. Questionnaires were administered every 6 months. Outcomes were presenteeism [Rheumatoid Arthritis Work Instability Scale (RA-WIS)], time to WC $\geq$ 6 months, and time to WC $\geq 2$ months (secondary outcome). Baseline characteristics (age, gender, ethnicity, occupation, education, disease duration and self-employment) were collected. Intention-to-treat (ITT) Iongitudinal analysis of RA-WIS using linear mixed effect regression models with 2-year comparison as primary endpoint and survival analysis for time to WC using Kaplan-Meier and Cox Proportional Hazard models were performed. Robustness analyses were conducted by using various missing values imputation methods like last observation carried forward, imputation using worse possible outcomes and model-based multiple imputations; using square root transformation of RA-WIS outcome; and adjusting for baseline covariates. SAS version 9.4 was used.

Results: A total of 564 participants were recruited, with 478 (84.75\%) completing 2-year follow-up. Baseline characteristics were similar between groups Mean RA-WIS scores were significantly lower in the intervention group from 6 months onwards, with the greatest difference observed at 2 years $(-1.78$ $95 \% \mathrm{Cl}:-2.7,-0.9, \mathrm{p}<.0001$ ), yielding a standardized effect size of $32 \%$. Satisfactory robustness was observed. Work cessation occurred less often in intervention than control groups, but only reached statistical significance for WC $\geq$ 2 months (WC $\geq 6$ months: 31 versus 44 events, aHR $0.70,95 \% C l: 0.44,1.11$, $\mathrm{p}=0.13 ; \mathrm{WC} \geq 2$ months: 39 versus 61 events, aHR: $0.65,95 \% \mathrm{Cl}: 0.43,0.98$, $\mathrm{p}=0.04$ ).

Conclusion: Results of the RCT reveal the program was effective at improving presenteeism and preventing short-term WC. Effectiveness at preventing longterm work disability will be assessed at 5 years. This program fills one of the most important and costly unmet needs for people with inflammatory arthritis.

[1] Carruthers EC, Rogers P, Backman CL, et al. "Employment and arthritis: making it work" a randomized controlled trial evaluating an online program to help people with inflammatory arthritis maintain employment (study protocol). BMC Med Inform Decis Mak. 2014;14:59. Published 2014 Jul 21. doi:10.1186/1472-6947-14-59 
Figure 1. Mean RA-WIS by allocation group over 2 years of follow-up. Mean \pm Standard Error are plotted.

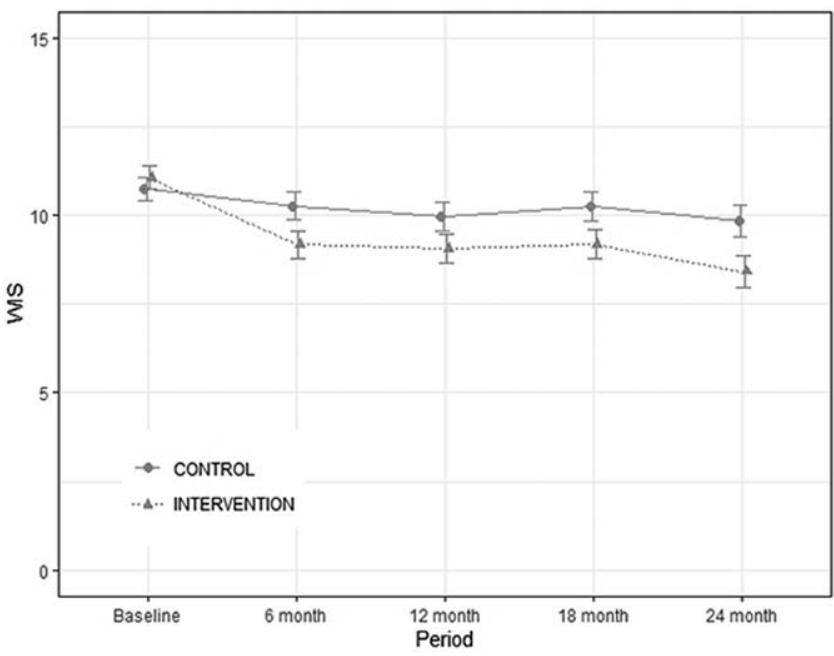

Figure 2. Kaplan Meier analysis of time to work cessation $\geq 2$ months

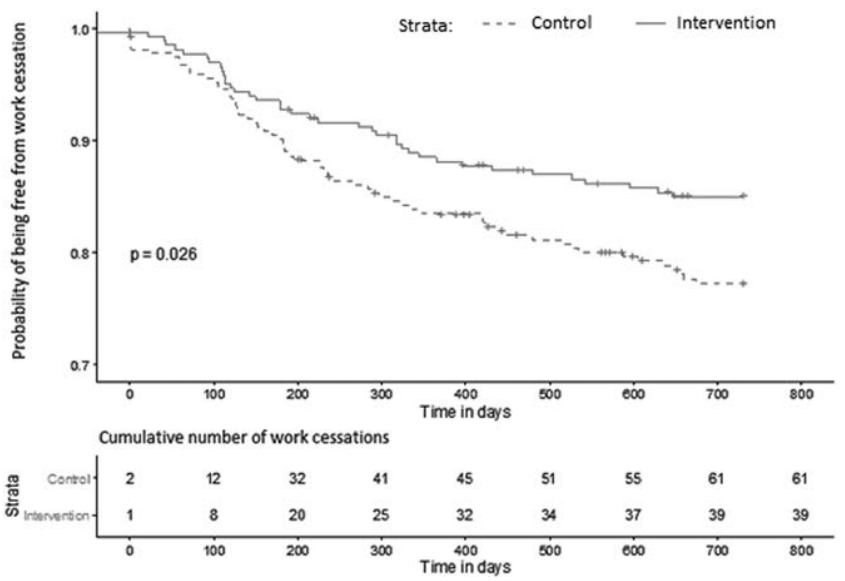

Disclosure of Interests: Andre Luquini: None declared, Yufei Zheng: None declared, Hui Xie: None declared, Catherine Backman: None declared, Pamela Rogers: None declared, Alex Kwok: None declared, Astrid Knight: None declared, Monique Gignac: None declared, Dianne Mosher: None declared, Linda Li: None declared, John Esdaile: None declared, Carter Thorne Consultant of: Abbvie, Centocor, Janssen, Lilly, Medexus/Medac, Pfizer, Speakers bureau: Medexus/ Medac, Diane Lacaille: None declared

DOI: 10.1136/annrheumdis-2020-eular.2383

\section{OP0011 A RANDOMIZED, DOUBLE-BLIND, ACTIVE- CONTROLLED STUDY OF AVACOPAN IN ANTI-NEUTROPHIL CYTOPLASMIC ANTIBODY (ANCA)-ASSOCIATED VASCULITIS}

P. A. Merkel ${ }^{1}$, D. Jayne ${ }^{2}$, H. Yue ${ }^{3}$, T. Schall ${ }^{3}$, C. Kelleher ${ }^{3}$, P. Bekker ${ }^{3}$ on behalf of the ADVOCATE Study Group. ${ }^{1}$ University of Pennsylvania, Rheumatology, Philadelphia, United States of America; ${ }^{2}$ University of Cambridge, Medicine, Cambridge, United Kingdom; ${ }^{3}$ ChemoCentryx, Inc., Mountain View, United States of America

Background: Complement fragment $\mathrm{C} 5 \mathrm{a}$ is strongly linked to the pathogenesis of Anti-Neutrophil Cytoplasmic Antibody (ANCA)-associated vasculitis (AAV). $\mathrm{C} 5 \mathrm{a}$ receptor $(\mathrm{C} 5 \mathrm{aR})$, present on neutrophils, is a G protein-coupled receptor for C5a. Avacopan (previously CCX168) is an orally-administered selective antagonist of C5aR which blocks C5a-induced cell activation. Two previous Phase 2 clinical trials provided evidence of effectiveness of avacopan in AAV and its potential to eliminate extensive use of glucocorticoids (GC) and GC-related toxicities.

Objectives: This Phase 3 study evaluated the efficacy and safety of avacopan for the treatment of AAV.
Methods: Eligible subjects were randomized 1:1 to receive either prednisone or avacopan in combination with either a) cyclophosphamide (oral or IV) followed by azathioprine or b) rituximab (four IV infusions). Randomization was stratified by the treatment regimen (rituximab, IV cyclophosphamide, or oral cyclophosphamide), ANCA serotype, and newly-diagnosed or relapsing disease. Treatment period was 52 weeks; primary efficacy endpoints were the proportion of subjects achieving disease remission at Week 26, and sustained disease remission at Week 52. Remission was defined as a Birmingham Vasculitis Activity Score (BVAS) of zero and not taking glucocorticoids for AAV within 4 weeks prior to Week 26. Sustained remission was defined as being in remission at Week 26 and also subsequently in remission as defined above at Week 52. Any relapse of AAV between Weeks 26 and 52 was considered not achieving sustained remission. Results: 330 subjects were randomized and dosed: 166 in avacopan and 164 in prednisone arms. At Week $26,72.3 \%$ subjects achieved remission in the avacopan arm compared to $70.1 \%$ in the prednisone arm ( $p<0.0001$ for non-inferiority) At Week 52, 65.7\% subjects achieved sustained remission in the avacopan arm compared to $54.9 \%$ in the prednisone arm achieving both non-inferiority and superiority to prednisone $\operatorname{arm}(\mathrm{p}=0.0066$ for superiority of avacopan).

The avacopan arm had a significant reduction in glucocorticoid-related toxicity compared to the prednisone arm as measured by the Glucocorticoid Toxicity Index (GTI) of Cumulative Worsening Score $(\mathrm{p}=0.0002)$ and Aggregate Improvement Score $(p=0.0082)$.

In subjects with renal disease at baseline, the avacopan arm showed a mean increase in estimated glomerular filtration rate (eGFR) of $7.3 \mathrm{~mL} / \mathrm{min} / 1.73 \mathrm{~m} 2$ from baseline to week 52 as compared to $4.0 \mathrm{~mL} / \mathrm{min} / 1.73 \mathrm{~m} 2$ increase in the prednisone arm $(\mathrm{p}=0.0259)$.

Overall subject incidence of serious adverse events (SAEs) was generally consistent with previous AAV trials at $45.1 \%$ and $42.2 \%$ for prednisone and avacopan groups, respectively. Serious infections were $15.2 \%$ and $13.3 \%$, serious hepatic adverse events $3.7 \%$ vs $5.4 \%$, and SAEs of white blood cell count decreases were $4.9 \%$ vs $2.4 \%$ for prednisone and avacopan, respectively. No meningococcal infections were reported.

Conclusion: Avacopan treatment resulted in remission in AAV patients receiving rituximab or cyclophosphamide/azathioprine at a rate that was non-inferior to the active comparator prednisone at week 26 and superior to prednisone in sustained remission at Week 52 . A significant reduction in glucocorticoid-related toxicity was observed in the avacopan vs. prednisone arms. Significant increase in eGFR in subjects with renal disease was also observed in avacopan vs. prednisone. The safety profile of avacopan appears acceptable for development in AAV. Avacopan treatment for AAV is efficacious and exhibits benefits not seen with chronic prednisone therapy.

References: None.

Disclosure of Interests: Peter A Merkel Grant/research support from: AstraZeneca, Boeringher-Ingelheim, Bristol-Myers Squibb, Celgene, ChemoCentryx, Forbius, Genentech/Roche, Genzyme/Sanofi, GlaxoSmithKline, InflaRx Kypha, TerumoBCT, Consultant of: Abbvie, AstraZeneca, Biogen, Boeringher-Ingelheim, Bristol-Myers Squibb, Celgene, ChemoCentryx, CSL Behring, For bius, Genentech/Roche, Genzyme/Sanofi, GlaxoSmithKline, InflaRx, Insmed, Jannsen, Kiniksa, Pfizer, Sparrow, Talaris, David Jayne Grant/research support from: ChemoCentryx, GSK, Roche/Genentech, Sanofi-Genzyme, Consultant of: Astra-Zeneca, ChemoCentryx, GSK, InflaRx, Takeda, Insmed, Chugai Boehringer-Ingelheim, Huibin Yue Shareholder of: ChemoCentryx, Employee of: ChemoCentryx, Thomas Schall Shareholder of: ChemoCentryx, Employee of: ChemoCentryx, Catherine Kelleher Shareholder of: Amgen, ChemoCentryx, Inc., Grant/research support from: Not active, Consultant of: Independent Consultant in the past, Employee of: ChemoCentryx, Inc., Pirow Bekker Shareholde of: Stock options ChemoCentryx, Consultant of: ChemoCentryx, Employee of: ChemoCentryx

DOI: 10.1136/annrheumdis-2020-eular.1073

\section{\begin{tabular}{|l|l|l|l|}
\hline OP0012 & TNF INHIBITORS ARE ASSOCIATED WITH A REDUCED
\end{tabular} RISK OF VENOUS THROMBOEMBOLISM COMPARED TO CSDMARDS IN RA PATIENTS}

M. Schaefer ${ }^{1}$, M. Schneider ${ }^{2}$, A. Graessler ${ }^{3}$, W. Ochs ${ }^{4}$, A. Zink ${ }^{1}$, A. Strangfeld ${ }^{1}$ ${ }^{1}$ German Rheumatism Research Centre, Berlin, Germany; ${ }^{2}$ Scientific Advisory Board, Duesseldorf, Germany; ${ }^{3}$ Rheumatologist, Pirna, Germany; ${ }^{4}$ Rheumatologist, Bayreuth, Germany

Background: While the short-term use of bDMARDs up to 180 days has been associated with an increased risk of venous thromboembolism (VTE) compared to csDMARDs in patients with rheumatoid arthritis (RA), the long term use of more than 730 days has been associated with a decreased risk based on claims data [1]. Among patients with inflammatory bowel disease, observational data indicated that TNF inhibitors may have a protective effect regarding the VTE risk [2]. 